\title{
Resonant radiation shed by dispersive shock waves
}

\author{
Matteo Conforti, ${ }^{1}$ Fabio Baronio, ${ }^{1}$ and Stefano Trillo ${ }^{2}$ \\ ${ }^{1}$ Dipartimento di Ingegneria dell' Informazione, Università di Brescia, Via Branze 38, 25123 Brescia, Italy \\ ${ }^{2}$ Dipartimento di Ingegneria, Università di Ferrara, Via Saragat 1, 44122 Ferrara, Italy
}

(Received 5 May 2013; published 9 January 2014)

\begin{abstract}
We analyze resonant radiation emitted by dispersive shock waves owing to higher-order dispersive corrections of the leading term in the defocusing nonlinear Schrödinger equation. We give criteria for calculating the radiated frequency based on an analytical estimate of the shock velocity and reveal a diversity of scenarios controllable via the corrections, ranging from the radiation-induced transition of the dispersive shock into a classical-type shock to the qualitative modification of the underlying gradient catastrophe or the competition between different breaking mechanisms.
\end{abstract}

DOI: 10.1103/PhysRevA.89.013807

PACS number(s): 42.65.Sf, 42.65.Re, 47.40.Nm, 52.35.Tc

\section{INTRODUCTION}

Dispersive shock waves (DSWs) are expanding regions filled with fast oscillations that stem from the dispersive regularization of classical shock waves (SWs). Originally introduced in collisionless plasmas [1] and water waves [2], it is only recently that they have been the focus of intense multidisciplinary efforts that have established their universal role in atom condensates [3,4], light pulse (temporal) [5] and beam (spatial) [6] propagation, oceanography [7], quantum liquids [8], electron beams [9], magma flow [10], granular materials [11], and wave or material disorder [12]. The dynamics of DSWs is understood in terms of a weakly dispersive formulation of integrable models (and their deformations) such as the Korteweg-De Vries [1,9], the Benjamin-Ono [8,12,13], or the defocusing nonlinear Schrödinger equation (dNLSE) [4-6,12,14]. However, since the leading-order dispersion of such models must be extremely weak for the phenomenon to take place, one is naturally led to wonder about the effects of higher-order dispersion (HOD), which must be accounted for to describe the actual dispersion in many physical situations. The aim of this work is to show that HOD corrections lead DSWs to emit resonant radiation (RR) due to a specific phase matching with linear waves, which can ultimately alter the shock dynamics itself.

The emission of RR is usually thought to be a prerogative of solitons [15-25]. The spectacular phenomenon of optical supercontinuum generation has offered the possibility to highlight the role of RR for perturbed solitons of the focusing NLSE (fNLSE) [19-21]. While soliton-driven RR is generally understood also thanks to recent studies of new regimes (tapered [22] and noble-gas-filled fibers [23], frequency combs [24], slowlight waveguides [25], and quadratic media [26]) or new paradigms (negative-frequency RR [27]), the problem of RR from SWs was overlooked. Here we show that perturbed DSWs emit RR, owing to the strong spectral broadening that accompanies wave breaking, which seeds linear waves that are resonantly amplified thanks to the well-defined velocity of the shock front. While we expect the mechanism of RR from perturbed dispersive shocks to be universal and observable in different physical contexts, we specifically formulate our approach with reference to temporal pulse propagation ruled by the dNLSE [28], which has immediate application to optical fibers pumped in the normal group-velocity-dispersion regime [5,29-31]. In this regime recent experiments have pointed out the occurrence of RR phenomena [30], which can also have direct impact over the broadband spectral feature related to the type of supercontinuum developing in the normal dispersion regime [29]. In particular, as recently shown by some of us [31], the results of Ref. [30] can be correctly interpreted in terms of RR from pulse wave breaking, further enhanced by the Raman response of the fiber. However, neither does the zero-pulse background considered in Refs. [30,31] give the most favorable condition for the full development of the dispersive shock, nor are the dynamics of the radiative process limited to the scenario discussed therein. This calls for an exhaustive analysis of the radiative phenomenon, which is the main aim of this paper. In particular, our purpose here is to investigate, in the framework of a model of general interest (namely, the dNLSE with HOD corrections), the qualitatively different radiative processes that can take place depending on the order and magnitude of HOD terms. We point out, in particular, at possible cross-over scenarios induced by increasing the leading-order HOD (with special attention to the calculation of the relevant shock velocity), the modification of the breaking process itself, and the role of the competing instability processes.

The paper is organized as follows: In Sec. II we recall the origin of the semiclassical generalized dNLSE model and analyze the phase-matching condition for resonance between linear waves and a nonlinear pump. In Sec. III, we derive the generalized dispersionless (hydrodynamic) limit of the starting model and discuss the notion of shock velocity. Section IV is devoted to discuss all the possible RR scenario determined by the leading terms (third-order and fourth-order dispersion) in the perturbation. The details of the calculations regarding the dispersionless model and the calculation of the shock leading edge velocity in the framework of Whitham equations are reported in Appendixes A and B, respectively.

\section{RESONANT RADIATION RULED BY NONLINEAR SCHRÖDINGER EQUATION IN SEMICLASSICAL REGIME}

We consider the dNLSE obeyed in the laboratory frame by a slowly varying envelope $A(Z, T)$ with central frequency $\omega_{0}$ and wave number $k_{0}=k\left(\omega_{0}\right)$. The dNLSE which arises from the standard expansion of the wave number $k(\omega)$ around $\omega_{0}$, once expressed in terms of the real-world all-order dispersion 
operator $d_{T}\left(\partial_{T}\right)=\sum_{n \geqslant 1} \partial_{\omega}^{n} k\left(i \partial_{T}\right)^{n} / n$ ! (all the derivatives being implicitly evaluated at $\left.\omega_{0}\right)$, reads as

$$
i \partial_{Z} A+d_{T}\left(\partial_{T}\right) A+\gamma|A|^{2} A=0 .
$$

Note that the defocusing feature arises from the assumption $\partial_{\omega}^{2} k>0$ (normal dispersion), assuming also $\gamma>0$. In order to make it clear that we operate in the weakly dispersive regime, it is convenient to cast Eq. (1) in semiclassical form. To this end we introduce the retarded time $T^{\prime}=T-Z / V_{g}$ in the frame traveling at the natural group velocity $V_{g}=\left(\partial_{\omega} k\right)^{-1}$ and scale $T^{\prime}$ to the input pulse width $T_{0}$ and the distance $Z$ to the geometric mean $Z_{0}=\sqrt{L_{n l} L_{d}}$, introducing $z=$ $Z / Z_{0}$ and $t=T^{\prime} / T_{0}$. Here $L_{d}=T_{0}^{2} / \partial_{\omega}^{2} k$ is the characteristic dispersive length associated with second-order dispersion $\partial_{\omega}^{2} k$, and $L_{n l}=(\gamma P)^{-1}$ is the characteristic nonlinear length associated with the input peak power $P$ and nonlinear coefficient $\gamma$. Defining also the field $\psi=A / \sqrt{P}$ [accordingly, in the numerics, we have $\max [\psi(z=0, t)]=1]$, the smallness parameter $\varepsilon=\sqrt{L_{n l} / L_{d}}$, and the dispersion coefficients $\beta_{n}=$ $\partial_{\omega}^{n} k /\left[\left(L_{n l}\right)^{n-2}\left(\partial_{\omega}^{2} k\right)^{n}\right]^{1 / 2}$ (note that, by definition, $\beta_{2}=1$ ), we recover the dNLSE in the weakly dispersive form (henceforth sum over $n$ implicitly assumes $n \geqslant 2$ ):

$$
\begin{aligned}
i \varepsilon \partial_{z} \psi & +d\left(\partial_{t}\right) \psi+|\psi|^{2} \psi=0 \\
d\left(\partial_{t}\right)= & \sum_{n} \frac{\beta_{n}}{n !} \varepsilon^{n}\left(i \partial_{t}\right)^{n}=-\frac{\varepsilon^{2}}{2} \partial_{t}^{2}-i \frac{\beta_{3} \varepsilon^{3}}{6} \partial_{t}^{3} \\
& +\frac{\beta_{4} \varepsilon^{4}}{24} \partial_{t}^{4}+\cdots .
\end{aligned}
$$

Note that the normalized dispersive operator $d\left(\partial_{t}\right)$ has progressively smaller terms, weighted by powers of the parameter $\varepsilon \ll 1$ and coefficients $\beta_{n}$.

We assume an input pump $\psi_{0}=\psi(t, z=0)$ with central frequency $\omega_{p}=0$ [i.e., in real-world units $\omega_{p}$ coincides with $\omega_{0}$, around which $d\left(\partial_{t}\right)$ in Eq. (2) is expanded]. Let us denote as $V_{s}$ the "velocity" of the SW near a wave-breaking point (note that, here, $V_{s}=d t / d z$ is the reciprocal of the velocity as usually defined for soliton RR [16]) and denote as $\tilde{d}(i \omega)$ the Fourier transform of $d\left(\partial_{t}\right)$. Linear waves $\exp [i k(\omega) z-i \omega t]$ are resonantly amplified when their wave number in the shockmoving frame, which reads as $k(\omega)=\frac{1}{\varepsilon}\left[\tilde{d}(i \omega)-V_{s}(\varepsilon \omega)\right]$ equals the pump wave number $k_{p}=k\left(\omega_{p}=0\right)=0$. Denoting also as $k_{n l}$ the difference between the nonlinear contributions to the pump and RR wave number [32], respectively, the radiation is resonantly amplified at frequency detuning $\omega=\omega_{R R}$ that solves the explicit equation

$$
\sum_{n} \frac{\beta_{n}}{n !}(\varepsilon \omega)^{n}-V_{s}(\varepsilon \omega)=\varepsilon k_{n l} .
$$

We show below that Eq. (3) correctly describes the RR emitted by a DSW. At variance with solitons of the fNLSE where $V_{s}\left(\omega_{p}=0\right)=0[16,20]$, DSWs possess nonzero velocity $V_{s}$, which must be carefully evaluated, having great impact on the determination of $\omega_{R R}$.

\section{DISPERSIVE SHOCKS IN DEFOCUSING NONLINEAR SCHRÖDINGER EQUATION}

The process of wave breaking ruled by Eq. (2) can be described by applying the Madelung transformation $\psi=$ $\sqrt{\rho} \exp (i S / \varepsilon)$. At leading order in $\varepsilon$, we obtain a quasilinear hydrodynamic reduction, with $\rho=|\psi|^{2}$ and $u=-S_{t}$ being equivalent density and velocity of the flow, respectively, which can be further cast in the form

$$
\begin{gathered}
\partial_{z} \rho+\partial_{t}\left[\sum_{n} \frac{\beta_{n}}{(n-1) !}\left(\rho u^{n-1}\right)\right]=0, \\
\partial_{z}(\rho u)+\partial_{t}\left[\sum_{n} \frac{\beta_{n}}{(n-1) !} \rho u^{n}+\frac{1}{2} \rho^{2}\right]=0,
\end{gathered}
$$

of a conservation law $\partial_{z} \mathbf{q}+\partial_{t} \mathbf{f}(\mathbf{q})=0$ for mass and momentum (see Appendix A), with $\mathbf{q}=(\rho, \rho u)$. This system can be also conveniently diagonalized to yield

$$
\partial_{z} r^{ \pm}+V^{ \pm} \partial_{t} r^{ \pm}=0
$$

by introducing the eigenvelocities $V^{ \pm}=\sum_{n} \beta_{n} u^{n-1} /$ $(n-1) ! \pm\left[\rho \sum_{n} \beta_{n} u^{n-2} /(n-2) !\right]^{1 / 2}$ and the Riemann invariants $r^{ \pm}=u \pm 2 \sqrt{\rho}\left[\sum_{n} \beta_{n} u^{n-2} /(n-2) !\right]^{-1 / 2}$.

Equations (4) and (5), as far as HOD is such that they remain hyperbolic, admit weak solutions in the form of classical SWs, i.e., traveling discontinuity from left $\left(\rho_{l}, u_{l}\right)$ to right $\left(\rho_{r}, u_{r}\right)$ values, whose velocity $V_{c}$ can be found from the so-called Rankine-Hugoniot $(\mathrm{RH})$ condition $V_{c}\left(\mathbf{q}_{l}-\mathbf{q}_{r}\right)=$ $\left[\mathbf{f}\left(\mathbf{q}_{l}\right)-\mathbf{f}\left(\mathbf{q}_{r}\right)\right]$ [33]. In the $2 \times 2$ case, the RH equations fix both $V_{c}$ and the admissible value of one of the parameters of the jump, e.g., $u_{r}$ given $\rho_{r}, \rho_{l}, u_{l}$. For instance, when no HOD is effective (take $\beta_{2}=1$ ), an admissible right-going shock which satisfies the entropy condition $\rho_{l}>\rho_{r}$, can be obtained with

$$
\begin{aligned}
& u_{r}=u_{l}-\left(\rho_{l}-\rho_{r}\right) \sqrt{\frac{\rho_{r}+\rho_{l}}{2 \rho_{l} \rho_{r}}}, \\
& V_{c}=u_{l}+\rho_{r} \sqrt{\frac{\rho_{r}+\rho_{l}}{2 \rho_{l} \rho_{r}}} .
\end{aligned}
$$

This result can be generalized for HOD, thanks to Eqs. (4) and (5). For instance, if $\beta_{3} \neq 0$, the SW velocity becomes

$$
V_{c}=\frac{\beta_{2}\left(\rho_{l} u_{l}-\rho_{r} u_{r}\right)+\beta_{3}\left(\rho_{l} u_{l}^{2}-\rho_{r} u_{r}^{2}\right) / 2}{\left(\rho_{l}-\rho_{r}\right)},
$$

where $u_{r}$ is obtained as the real root of the cubic equation $\beta_{3}\left(u_{l}-u_{r}\right)^{2}\left(u_{l}+u_{r}\right)+2 \beta_{2}\left(u_{l}-u_{r}\right)^{2}=g\left(\rho_{l}, \rho_{r}\right)$, where $g\left(\rho_{l}, \rho_{r}\right) \equiv\left(\rho_{l}-\rho_{r}\right)^{2}\left(\rho_{r}+\rho_{l}\right) /\left(\rho_{l} \rho_{r}\right)$ (see Appendix A).

Second-order dispersion, however, is known to regularize classical SWs by replacing the jump with an expanding fan filled with oscillations described in terms of a modulated nonlinear periodic wave. In this case the SW velocity $V_{c}$ is replaced by the velocities of the leading $V_{l}$ and trailing $V_{t}$ edges (with $V_{l}<V_{c}<V_{t}$ ), where the periodic wave locally tends to a soliton and a linear wave, respectively. HOD induces this structure to radiate, also altering the dynamics of SW formation. In the following we specifically focus on the effect of two leading HOD, namely third-order (3-HOD) and fourth-order (4-HOD) dispersion, showing how RR is shed 
with different features depending on the specific dispersive correction.

\section{RESONANT RADIATION EMITTED BY DISPERSIVE SHOCKS}

\section{A. Third-order dispersion}

When 3-HOD is effective we find a crossover from a perturbative regime $\left(\left|\beta_{3}\right| \lesssim 0.5\right)$ where the DSW leading edge turns out to be responsible for the $\mathrm{RR}$, to a regime where the 3-HOD is strong enough $\left(\left|\beta_{3}\right| \sim 1\right)$ to modify the shock formation, leading to enhanced RR produced by a traveling front which is approximated with a classical SW. To show this and verify that Eq. (3) is able to predict the RR frequency in both regimes, we consider first a step initial value that allows us to calculate analytically the velocity. Without loss of generality, we take $\beta_{3}<0$. Specifically, we consider the evolution of an initial jump from the "left" state $\rho_{l}, u_{l}=0$ for $t<0$ to the "right" state $\rho_{r}\left(<\rho_{l}\right), u_{r}=$ $2\left(\sqrt{\rho_{r}}-\sqrt{\rho_{l}}\right)$ for $t>0$, which is such to maintain constant $r^{-}(z=0, t)$ while $r^{+}(z=0, t)$ has step-like variation. In this case, the modulated wave train produced upon evolution [see Figs. 1(a) and 1(c)] in the limit $\beta_{3}=0$, is described by a rarefaction wave of the Whitham modulation equations for the unperturbed dNLSE [4]. Following the approach of Ref. [4] and exploiting the fact that only one Riemann variable of such Whitham equations changes, one can calculate the edge velocities of the fan (see Appendix B). What is relevant for the RR is the leading-edge velocity, which we find to be $V_{l}=\sqrt{\rho_{l}}+u_{r}=2 \sqrt{\rho_{r}}-\sqrt{\rho_{l}}$ (note that this differs from the result of Ref. [4] because we choose a different step-like initial value with $u_{l}=0$ and $\rho_{r} \neq 1$ ). Given a gray soliton on an unchirped background $A, \psi=A[w \tanh (\theta)+i v]$
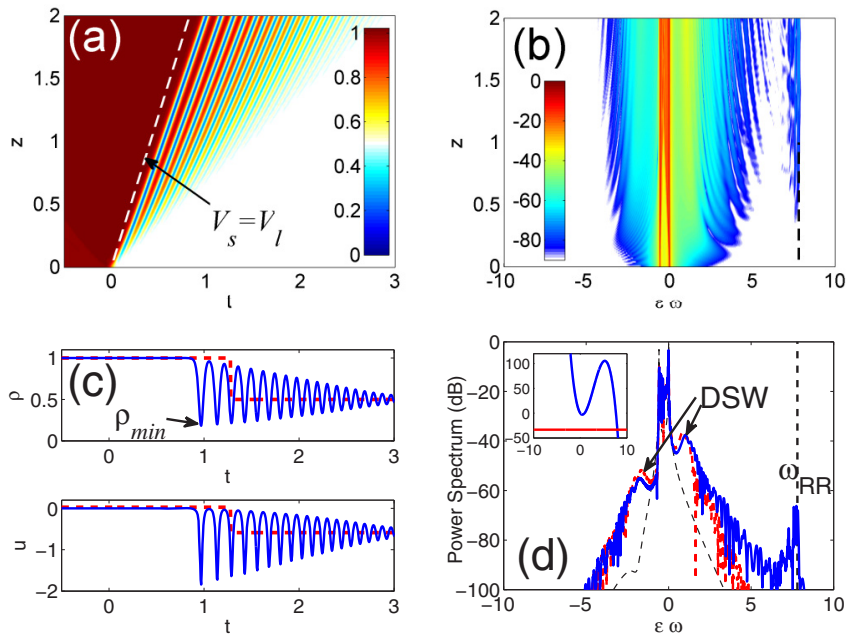

FIG. 1. (Color online) Radiating DSW from dNLSE (2) with $\varepsilon=0.03$, input step $\rho_{l}, \rho_{r}=1,0.5$, and 3-HOD $\beta_{3}=-0.35$ : (a) Color-level plot of density $\rho(t, z)$ (the dashed line gives the DSW leading edge velocity $V_{l}$ ); (b) corresponding spectral evolution; (c) snapshots at $z=2$ of $\rho, u$ of unperturbed case $\beta_{3}=0$ (in dashed red is the corresponding classical SW); (d) comparison of output spectra with (thick solid blue line) and without (dashed red) 3-HOD (input thin dashed line). Inset shows graphical solution of Eq. (3). $\exp \left(i A^{2} z / \varepsilon\right), \theta=\frac{w}{\varepsilon}(t-A v z), w^{2}=1-v^{2}, V_{l}$ turns out to coincide with the soliton velocity $V_{\text {sol }}=A v=\sqrt{\rho_{\text {min }}}$, with natural position $A=\sqrt{\rho_{l}}, v=\left(2 \sqrt{\rho_{r}}-\sqrt{\rho_{l}}\right) / \sqrt{\rho_{l}}$, and the dip density $\rho_{\min }=\left(2 \sqrt{\rho_{r}}-\sqrt{\rho_{l}}\right)^{2}$. We emphasize, however, that the equivalence of the leading edge with a gray soliton holds only locally since the DSW is strictly speaking a modulated nonlinear wave.

In this regime, if we account for $k_{n l}=k_{n l}^{\text {sol }}-k_{n l}^{R R}=-\frac{1}{\varepsilon} \rho_{l}$ arising from the soliton $k_{n l}^{\text {sol }}=\rho_{l} / \varepsilon$ and the cross-induced contribution $k_{n l}^{R R}=2 \rho_{l} / \varepsilon$ to the RR, Eq. (3) explicitly reads

$$
\frac{\beta_{3}}{6}(\varepsilon \omega)^{3}+\frac{\beta_{2}}{2}(\varepsilon \omega)^{2}-V_{s}(\varepsilon \omega)+\rho_{l}=0 .
$$

Real solutions $\omega=\omega_{R R}$ of Eq. (9), with $V_{s}=V_{l} \equiv 2 \sqrt{\rho_{r}}-$ $\sqrt{\rho_{l}}$ correctly predicts the RR as long as $\left|\beta_{3}\right| \lesssim 0.5$, as shown by the dNLSE simulation in Fig. 1. The DSW displayed in Fig. 1(a) clearly exhibits a spectral RR peak besides spectral shoulders due to the oscillating front, as shown by the spectral evolution in Fig. 1(b) and the output spectrum (compared with the unperturbed one) in Fig. 1(d). Perfect agreement is found between the RR peak obtained in the numerics and the prediction [dashed vertical line in Figs. 1(b) and 1(d)] from Eq. (9) with velocity $V_{s}=V_{l}$ characteristic of the integrable limit $\left[\beta_{3}=0\right.$, snapshots in Fig. 1(c)]. Indeed, in this regime, the DSW leading edge is nearly unaffected by 3-HOD, whereas using the velocity $V_{c}$ [Eq. (7)] of the equivalent classical SW [reported for comparison in Fig. 1(c)] would miss the correct estimate of $\omega_{R R}$. We also point out that $k_{n l}$ represents a small correction, so $\omega_{R R}$ can be safely approximated by dropping the last term in Eq. (9) to yield

$$
\varepsilon \omega_{R R}=\frac{3}{2 \beta_{3}}\left(-\beta_{2} \pm \sqrt{\beta_{2}^{2}+8 V_{s} \beta_{3} / 3}\right),
$$

that can be reduced to the simple formula $\varepsilon \omega_{R R}=-3 \beta_{2} /$ $\beta_{3}$ [30] only when $\beta_{3} V_{s} \rightarrow 0$.

When $\left|\beta_{3}\right|$ grows larger, the aperture of the shock fan reduces (the difference between leading and trailing edge velocities decreases), until quite unexpectedly the DSW resembles a single traveling front, i.e., a classical SW [34]. In this regime, we find that Eq. (9) still gives the correct frequency $\omega_{R R}$ provided that $V_{S}$ is taken as the Rankine-Hugoniot velocity $V_{c}$ of the equivalent classical SW calculated for $\beta_{3} \neq 0$ [Eq. (8)] [35]. An example of this behavior is illustrated in Fig. 2 for $\beta_{3}=-1$. The RR becomes clearly visible in the temporal evolution [Fig. 2(a) and snapshots in Fig. 2(c)] and is sufficiently strong to generate also $-\omega_{R R}$ via four-wave mixing, as is clear from the spectrum [Figs. 2(b)-2(d)]. Perfect agreement between the numerics and the value predicted from Eq. (9), once we set $V_{s}=V_{c}$, is found also in this case. A physical interpretation of this remarkable transition from dispersive to classical shock is that the emitted radiation behaves as a local loss for the shock front.

The behaviors of step initial data are basically recovered for pulse waveforms that are more manageable in experiments. Figure 3 shows the transition from the perturbative [Fig. 3(a)] to the nonperturbative [Fig. 3(b)] regime, for an input gaussian pulse $\psi_{0}=v+(1-v) \exp \left(-t^{2}\right)$ with background-to-peakdensity ratio $v^{2}=0.09$. As shown in Fig. 3(a), for relatively small $\beta_{3}$, two asymmetric DSWs emerge from wave-breaking 

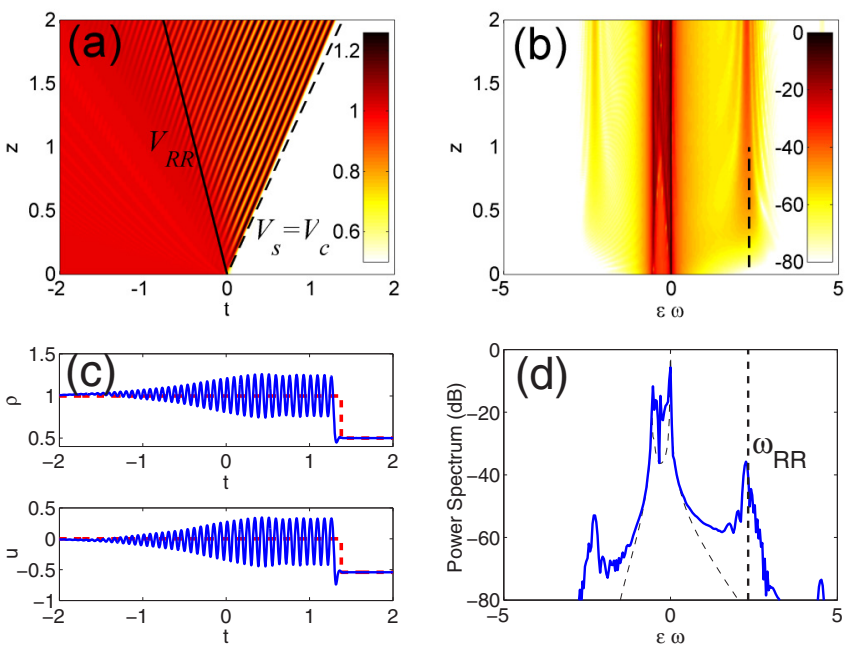

FIG. 2. (Color online) As in Fig. 1 with larger 3-HOD $\beta_{3}=-1$. The dashed vertical line in panels (b) and (d) gives $\omega_{R R}$ from Eq. (9) with velocity $V_{s}=V_{c}$ [corresponding to dashed line in panel (a)]. Here $V_{c}=0.69$ and $u_{r}=-0.543$ are the parameters of the classical SW [shown by dashed red line in panel (c)] from Eq. (8), see also Fig. 6 in Appendix A. Solid line in panel (a) indicates the velocity $V_{R R}$ of the RR.

points on the two pulse edges, which occur at different distances due to broken symmetry in time caused by 3-HOD. Phase matching is achieved only for the DSW traveling with $V_{s}>0$. The corresponding $\omega_{R R}$ can be obtained from Eq. (9) provided we set $V_{s}=V_{l}$, with the DSW leading-edge velocity being (following the discussion of Fig. 1) $V_{l}=\sqrt{\rho_{\min }}+u_{l}$, where the minimum density and the correction $u_{l}$ due to the local nonzero chirp are evaluated numerically after wave breaking as shown in Fig. 3(c). Indeed, these parameters cannot
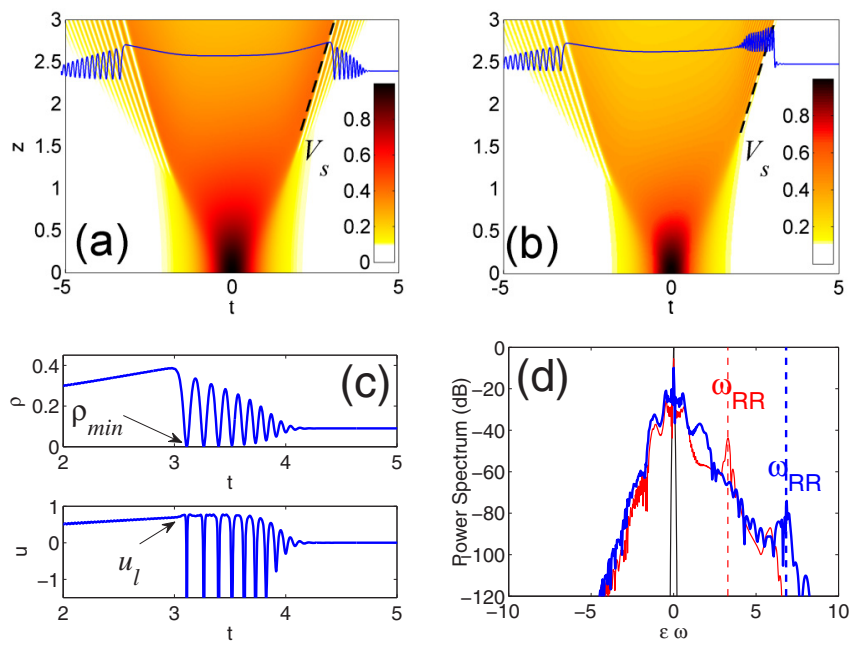

FIG. 3. (Color online) Radiating DSW from Gaussian pulses with small background $v^{2}=0.09$ : (a) $\beta_{3}=-0.35$. (b) $\beta_{3}=-0.6$. (c) Parameters determining the leading-edge velocity $V_{s}=\sqrt{\rho_{\min }}+$ $u_{l}=\sqrt{10^{-4}}+0.76=0.77$ [dashed line in panel (a)]; snapshot at $z=3$. (d) Output spectra (thick blue line, $\beta_{3}=-0.35$; thin red line, $\beta_{3}=-0.6$ ). The dashed lines give $\varepsilon \omega_{R R}$ calculated from Eq. (9). Here $\varepsilon=0.03$ and blue curves in panels (a) and (b) depict output snapshots.
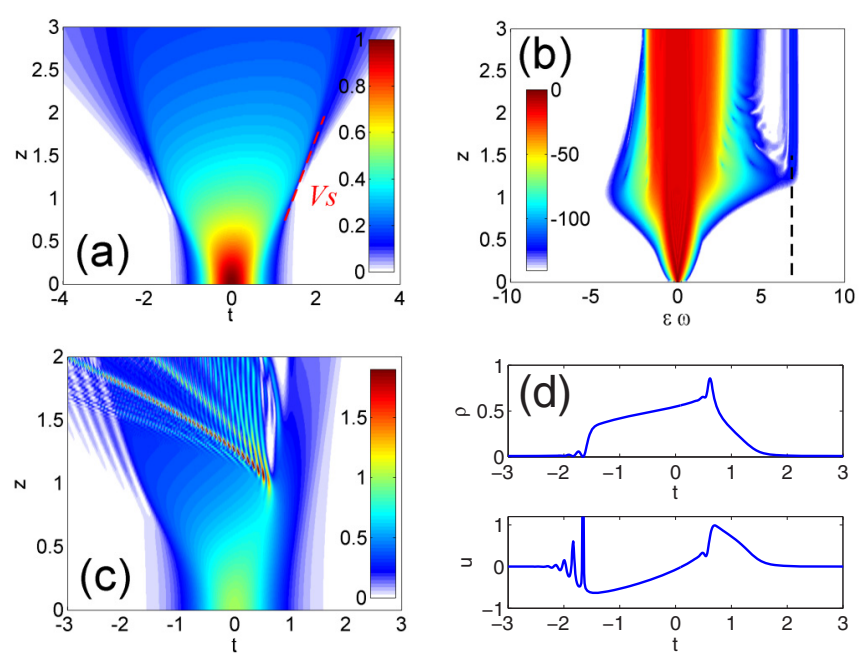

FIG. 4. (Color online) (a), (b) Temporal and spectral evolution of a Gaussian pulse without background emitting RR, for $\beta_{3}=-0.35$. (c), (d) Mixed hyperbolic-elliptic type of wave breaking for large 3 -HOD $\beta_{3}=-2, v^{2}=0.01$. Panel (d) reports a snapshot of $\rho$ and $u$ near the gradient catastrophe occurring at $z \approx 0.9$. Here $\varepsilon=0.03$.

be obtained analytically for a generic initial value problem, nor they are strictly constant upon evolution. Also in this case, a larger $\left|\beta_{3}\right|$ results in a narrower fan (and larger shock distance), until eventually a simple front is left which strongly radiates, as shown in Fig. 3(b). In this regime, a good approximation of the front velocity is obtained by the approximating classical SW in Eq. (8). In both the regimes shown in Figs. 3(a) and 3(b), Eq. (9) provides an accurate estimate of the RR frequency, as shown by the dashed lines reported in the output spectra in Fig. 3(d). Notice also that, for symmetry reasons, sign reversal of 3-HOD (i.e., $\beta_{3}>0$ ) simply results in RR with opposite frequency, generated by the DSW with opposite velocity $\left(V_{s}<0\right.$, left DSW).

We also emphasize two important points:

(i) As shown in Figs. 4(a) and 4(b), RR occurs also in the limit of vanishing background $v=0$, allowing us to conclude that a bright pulse does not need to be a soliton (as in the fNLSE, $\beta_{2}=-1$ ) to radiate. In fact, resonant amplification of linear waves occurs via SWs also in the opposite regime where the nonlinearity strongly enforces the effect of leading-order dispersion; the only key ingredients being a well-defined velocity of the front and the spectral broadening that seeds the RR at phase matching. Importantly, experimental evidence for such a RR scenario was reported very recently in fiber optics [30], without explaining the underlying mechanism, which our theory individuates in the shock formation. Indeed, the physical parameters used in Fig. 1 of Ref. [30], i.e., power $P=600 \mathrm{~W}$, pulse duration $T_{0}=1 \mathrm{ps}$, nonlinear coefficient $\gamma=2.5 \mathrm{~W}^{-1} \mathrm{~km}^{-1}$, dispersion $\partial_{\omega}^{2} k=7.5 \mathrm{ps}^{2} / \mathrm{km}$, $\partial_{\omega}^{3} k=0.2 \mathrm{ps}^{3} / \mathrm{km}$, gives normalized parameters $\varepsilon \simeq 0.07$ and $\beta_{3} \simeq 0.37$, typical of the wave-breaking regime $(\varepsilon \ll 1)$ with perturbative 3-HOD. Since $\beta_{3}>0$, the radiating shock turns out to be the one on the leading edge $(t<0)$, and its velocity $V_{s}=-0.75$, inserted into Eq. (3), gives a negative [opposite of Fig. 4(a)] frequency detuning $\Delta f_{R R}=\omega_{R R} T_{0}^{-1} /(2 \pi) \simeq$ $13 \mathrm{THz}$, in excellent agreement with the value reported in 
Ref. [30]. A detailed numerical study of this particular case, including Raman effects is reported in Ref. [31].

(ii) A limitation exists (regardless of $v$ ) on the value of $\left|\beta_{3}\right|$ to observe RR. Indeed, a large 3-HOD features a qualitatively different wave-breaking mechanism, as shown in Figs. 4(c) and 4(d) for $\beta_{3}=-2$. While the nonradiating (left) DSW simply develops at shorter $z$ without qualitative changes, on the right $(t>0)$ the pulse undergoes a different catastrophe, reminiscent of the fNLSE. Indeed, in this case the eigenvelocities $V^{ \pm}=\beta_{2} u+\beta_{3} u^{2} / 2 \pm \sqrt{\rho\left(\beta_{2}+\beta_{3} u\right)}$ become locally complex conjugate where $u>0$, implying that Eqs. (4) and (5) loose the hyperbolic character and are becoming elliptic (as in the fNLSE). This mixed-type behavior is reminiscent of a transonic flow (see, e.g., Ref. [36]) and leads to a different dynamics where RR plays no longer any role (this case will be studied in more detail elsewhere).

\section{B. Fourth-order dispersion}

A completely different scenario occurs when the dispersive correction is due to 4-HOD. In this case, the shock formation can compete with a different breaking mechanism [37]; namely, modulational instability (MI) which extends to the defocusing regime $\beta_{2}=1$, whenever $\beta_{4}<0$, as proven experimentally [38]. Although the MI analysis is known for this case [38], we report, for the convenience of the reader, the outcome of such analysis in our dimensionless units [Eq. (2)]. The continuous wave (cw) solution reads $\psi(z, t)=\sqrt{P_{0}} \exp \left[i z P_{0} / \varepsilon\right]$. MI arises when periodic perturbations in the form $p(z, t)=a(z) \exp [i \omega t]+b(z) \exp [-i \omega t]$ grow exponentially like $\exp [G z]$ at the expense of the $\mathrm{cw}$ pump. We find that the gain is $G(\varepsilon \omega)=|\operatorname{Im}[\lambda]| / \varepsilon$, where

$$
\lambda=\Sigma_{o} \pm \sqrt{\Sigma_{e}^{2}+2 P_{0} \Sigma_{e}},
$$

and $\quad \Sigma_{e}=\sum_{n \geqslant 1} \beta_{2 n}(\varepsilon \omega)^{2 n} /(2 n) ! \quad$ and $\quad \Sigma_{o}=\sum_{n \geqslant 1} \beta_{2 n+1}$ $(\varepsilon \omega)^{2 n+1} /(2 n+1)$ ! are the even and odd parts of dispersion, respectively. It is clear that only even dispersive terms determine the stability properties.

Considering 4-HOD $\beta_{4}<0 \quad\left(\beta_{2}=1\right)$, we have $\max G(\varepsilon \omega)=P_{0} / \varepsilon$ for

$$
\varepsilon \omega_{\text {peak }}=\sqrt{6 \frac{\beta_{2}+\sqrt{\beta_{2}^{2}+2 / 3\left|\beta_{4}\right| P_{0}}}{\left|\beta_{4}\right|}} .
$$

The gain band is $\varepsilon \omega \in\left[\varepsilon \omega_{\min }, \varepsilon \omega_{\max }\right]$ (and symmetric for $\omega \rightarrow-\omega)$, where

$$
\varepsilon \omega_{\min }=\sqrt{12 \frac{\beta_{2}}{\left|\beta_{4}\right|}}, \quad \varepsilon \omega_{\max }=\sqrt{6 \frac{\beta_{2}+\sqrt{\beta_{2}^{2}+4 / 3\left|\beta_{4}\right| P_{0}}}{\left|\beta_{4}\right|}} .
$$

Moreover, the phase-matching curve in Eq. (3), involving in this case a fourth-order polynomial, leads for the shock with $V_{s}>0$ to two possible phase-matching frequencies: $\omega_{R R 1}$ and $-\omega_{R R 2}\left(\omega_{R R 1,2}>0\right)$, which become four (two symmetric pairs) since opposite frequencies are phase matched by the shock with opposite velocity $V_{s}<0$ according to Eq. (3), as illustrated in Fig. 5(a). Our analysis shows that the two frequencies $\omega_{R R 1}$ and $\omega_{R R 2}$ (arising from shock on opposite edges) lie on the opposite sides of the MI gain curve
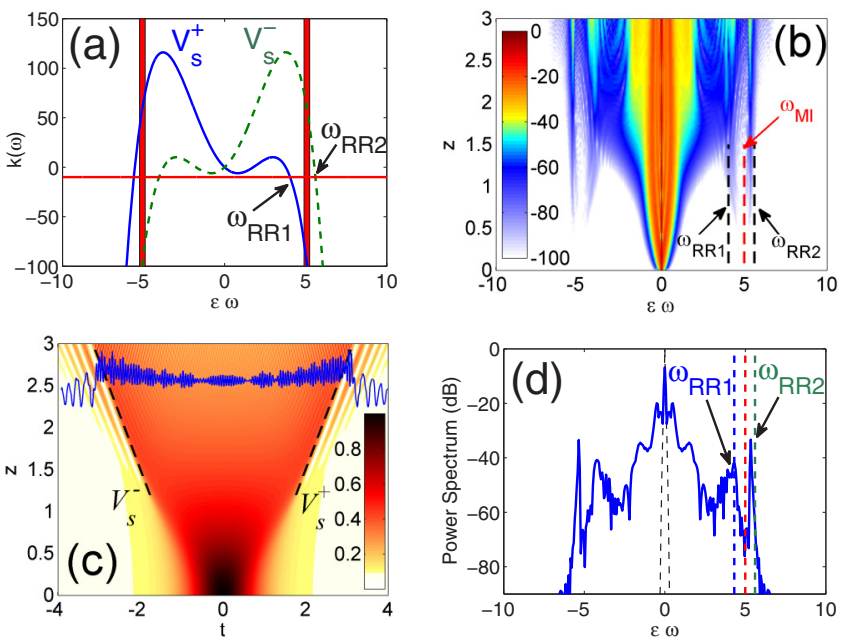

FIG. 5. (Color online) RR ruled by 4-HOD $\left(\beta_{3}=0, \beta_{4}=-0.5\right)$. (a) Dispersion curves from Eq. (3) for positive $\left(V_{s}^{+}\right.$, solid blue line) and negative ( $V_{s}^{-}$, dashed green line) velocity crossing $k_{n l}$ (horizontal red line) in $\pm \omega_{R R 1, R R 2}$; vertical stripes indicate MI gain bands. (b) Spectral evolution. (c) Temporal dynamics (blue curve depicts output snapshot). (d) Output spectrum [central red dashed line is peak MI gain, blue and green dashed lines correspond to RR frequencies obtained from panel (a)]. Here $\varepsilon=0.05$, input Gaussian pulse with background $v^{2}=0.09$.

(calculated for continuous waves), which has cutoff frequency $\varepsilon \omega_{c}=\sqrt{12 /\left|\beta_{4}\right|}$ and is narrow bandwidth. For an input pulse, $\mathrm{MI}$ amplifies frequencies in a larger bandwidth which serve as a seed for the RR. Indeed, as clear from the dNLSE simulation in Fig. 5(b), the twin-band RR starts to grow, triggered by MI, even during the process of pulse steepening [see evolution in Fig. 5(c)], while becoming prominent as the DSWs start to develop, traveling with definite velocities (here $V_{s}= \pm 0.77$ ). The RR frequencies from Fig. 5(a) fit well those reported in the numerical output spectrum in Fig. 5(d). The coexistence of the two wave-breaking phenomena (MI and DSW) is clearly visible in the output snapshot in Fig. 5(c).

\section{CONCLUSIONS}

We have demonstrated that dispersive shock waves resulting from the nonlinearity overbalancing a weak leading-order dispersion can emit resonant radiation owing to higher-order dispersive contributions. We have analyzed such phenomenon for the defocusing nonlinear Schrödinger equation, giving criteria for calculating the radiated frequency based on the estimate of the shock velocity and revealing also a diversity of possible scenarios depending on the order and magnitude of the dispersive corrections.

\section{ACKNOWLEDGMENT}

Funding from MIUR (Grants No. PRIN 2009P3K72Z and No. PRIN 2012BFNWZ2) is gratefully acknowledged.

\section{APPENDIX A}

Using the Madelung transformation $\psi=\sqrt{\rho} \exp (i S / \varepsilon)$ in Eq. (2), setting $u \equiv-\partial_{t} S$, we obtain at leading order the 
following system of quasilinear equations:

$$
\partial_{z}\left(\begin{array}{l}
\rho \\
u
\end{array}\right)+A \partial_{t}\left(\begin{array}{l}
\rho \\
u
\end{array}\right), \quad A=\left(\begin{array}{cc}
a_{11} & a_{12} \\
1 & a_{11}
\end{array}\right),
$$

where the matrix elements are

$$
\begin{aligned}
a_{11} & =\beta_{2} u+\frac{\beta_{3}}{2} u^{2}+\frac{\beta_{4}}{6} u^{3}+\frac{\beta_{5}}{24} u^{4}+\cdots \\
& =\sum_{n \geqslant 2} \frac{\beta_{n}}{(n-1) !} u^{n-1}, \\
a_{12} & =\rho\left(\beta_{2}+\beta_{3} u+\frac{\beta_{4}}{2} u^{2}+\frac{\beta_{5}}{6} u^{3}+\cdots\right) \\
& =\rho\left(\sum_{n \geqslant 2} \frac{\beta_{n}}{(n-2) !} u^{n-2}\right) .
\end{aligned}
$$

The eigenvalues of $A$ give the eigenvelocities $V^{ \pm}=a_{11} \pm \sqrt{a_{12}}$ of the diagonal form of Eqs. (A1) $\partial_{z} r^{ \pm}+V^{ \pm} \partial_{t} r^{ \pm}=0$, whereas the Riemann invariants $r^{ \pm}$can be easily found by standard techniques. By multiplying the first and the second of Eq. (A1), respectively, by $u$ and $\rho$ and summing up, we easily recover the equivalent form of a $2 \times 2$ conservation law $\partial_{z} \mathbf{q}+\partial_{t} \mathbf{f}(\mathbf{q})=0$ for $\mathbf{q}=(\rho, \rho u)$ [equivalent, in compact form, to Eqs. (4) and (5)]:

$$
\begin{gathered}
\partial_{z} \rho+\partial_{t}\left[\beta_{2} \rho u+\frac{\beta_{3}}{2} \rho u^{2}+\frac{\beta_{4}}{6} \rho u^{3}+\cdots\right]=0, \\
\partial_{z}(\rho u)+\partial_{t}\left[\beta_{2} \rho u^{2}+\frac{\beta_{3}}{2} \rho u^{3}+\frac{\beta_{4}}{6} \rho u^{4}+\cdots+\frac{1}{2} \rho^{2}\right]=0 .
\end{gathered}
$$

The velocity $V_{c}$ of a classical shock wave of Eqs. (A2) and (A3), can be obtained from Rankine-Hugoniot (RH) conditions

$$
V_{c}[[\mathbf{q}]]=[[\mathbf{f}(\mathbf{q})]],
$$

where $[[y]]=y_{l}-y_{r}$ indicates the "jump" of $y$ between left and right values. For instance, when we account for secondand third-order dispersion (the procedure can be iterated for higher-order dispersion), Eq. (A4) become explicitly

$$
\begin{aligned}
V_{c}\left(\rho_{l}-\rho_{r}\right)= & \beta_{2}\left(\rho_{l} u_{l}-\rho_{r} u_{r}\right)+\frac{\beta_{3}}{2}\left(\rho_{l} u_{l}^{2}-\rho_{r} u_{r}^{2}\right), \\
V_{c}\left(\rho_{l} u_{l}-\rho_{r} u_{r}\right)= & \beta_{2}\left(\rho_{l} u_{l}^{2}-\rho_{r} u_{r}^{2}\right)+\frac{\beta_{3}}{2}\left(\rho_{l} u_{l}^{3}-\rho_{r} u_{r}^{3}\right) \\
& +\frac{1}{2}\left(\rho_{l}^{2}-\rho_{r}^{2}\right) .
\end{aligned}
$$

By eliminating $V_{c}$ between these two equations, we arrive at the following equation:

$$
\beta_{3}\left(u_{l}-u_{r}\right)^{2}\left(u_{l}+u_{r}\right)+2 \beta_{2}\left(u_{l}-u_{r}\right)^{2}=g\left(\rho_{l}, \rho_{r}\right),
$$

where $g\left(\rho_{l}, \rho_{r}\right) \equiv\left(\rho_{l}-\rho_{r}\right)^{2}\left(1 / \rho_{r}+1 / \rho_{l}\right)$, which can be solved, for example, for $u_{r}$, once $\rho_{l}, \rho_{r}, u_{l}$ are assigned, while $V_{c}$ can be obtained by substituting back into one of the two RH equations, obtaining Eq. (8), which we repeat here for convenience:

$$
V_{c}=\frac{\beta_{2}\left(\rho_{l} u_{l}-\rho_{r} u_{r}\right)+\beta_{3}\left(\rho_{l} u_{l}^{2}-\rho_{r} u_{r}^{2}\right) / 2}{\left(\rho_{l}-\rho_{r}\right)} .
$$

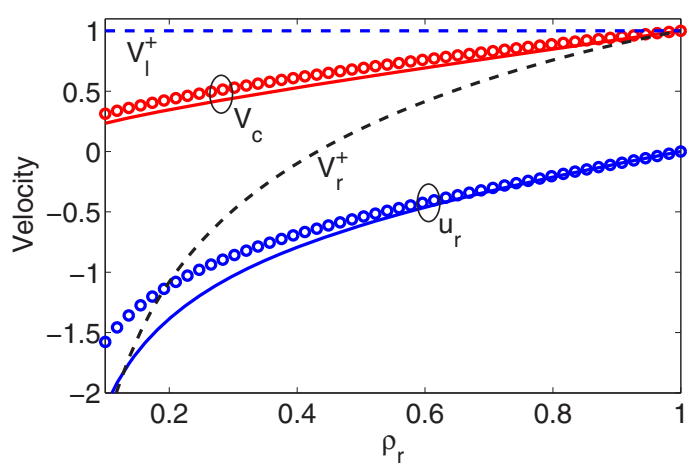

FIG. 6. (Color online) Right velocity $u_{r}$ and velocity $V_{c}$ of a weak SW solution for fixed left values $\rho_{l}=1, u_{l}=0$ and variable right density $\rho_{r}$ (i.e., variable amplitude of the jump), obtained from the RH conditions. We compare the case $\beta_{3}=0$ (solid curves) with $\beta_{3}=$ -1 (open circles). $V_{c}$ satisfies the entropy criterion $V_{l}^{+}>V_{c}>V_{r}^{+}$, where $V_{l}^{+}$and $V_{r}^{+}$(dashed curves) stand for the eigenvelocity $V^{+}$ calculated on the left and on the right of the jump for $\beta_{3} \neq 0$.

For $\beta_{3}=0$ we can easily solve Eqs. (A5) and (A6), obtaining

$$
\begin{aligned}
& u_{r}=u_{l} \mp\left(\rho_{l}-\rho_{r}\right) \sqrt{\frac{\rho_{r}+\rho_{l}}{2 \rho_{l} \rho_{r}}}, \\
& V_{c}=u_{l} \pm \rho_{r} \sqrt{\frac{\rho_{r}+\rho_{l}}{2 \rho_{l} \rho_{r}}} .
\end{aligned}
$$

We have thus two solutions, but only the one with upper sign satisfies the Lax entropy condition [39] for the right-going shock, i.e., the shock associated with the larger eigenvelocity $V^{+}$. The entropy condition states explicitly that the eigenvelocity must decrease across the discontinuity: $V_{l}^{+}>V_{c}>V_{r}^{+}$, where $V_{l, r}^{+}=V^{+}\left(\rho_{l, r}, u_{l, r}\right)$. This constraint along with the RH condition gives the well-known result that the physically admissible right-going shock wave requires $\rho_{l}>\rho_{r}$.

For $\beta_{3} \neq 0$ the formula becomes too cumbersome, so it turns out to be more practical to solve Eq. (A5) numerically. In this case we can again select the only physically admissible root by exploiting the entropy criterion. Figure 6 shows how $\beta_{3}$ modifies the velocity parameters $u_{r}$ and $V_{c}$.

\section{APPENDIX B}

In this appendix we outline the calculation of the leadingedge velocity of the DSW. Our approach closely follows that of Ref. [4], to which the reader is referred to for further details. Our aim here is to report only the essential formulas that lead to the expression of the leading-edge velocity of the DSW in our formulation of the initial step-like data.

Let us start by considering a traveling-wave periodic solution of the NLSE in the absence of HOD perturbations [i.e., Eq. (2) with $\beta_{n}=0, n \geqslant 3$ ] of the form $\psi(t, z)=$ $\sqrt{\rho(t-V z)} \exp [i \phi(\theta)]$, where $\theta \equiv \frac{t-V z}{\varepsilon}$ is a fast variable since $\varepsilon \ll 1$. By means of direct substitution into the NLSE, one can easily obtain the dn-oidal solution

$$
\rho(t, z)=\lambda_{3}-\left(\lambda_{3}-\lambda_{1}\right) \mathrm{dn}^{2}\left(\sqrt{\lambda_{3}-\lambda_{1}} \theta \mid m\right),
$$


which depends on the parameters $\lambda_{1}, \lambda_{2}, \lambda_{3}, V$, with the additional constraint $V=\sqrt{\lambda_{1}+\lambda_{2}+\lambda_{3}}$. Here the wave period $L=2 K(\mathrm{~m}) / \sqrt{\lambda_{3}-\lambda_{1}}$ is given in terms of the elliptic integral of the first kind $K(m)$, with $m=\left(\lambda_{2}-\lambda_{1}\right) /\left(\lambda_{3}-\lambda_{1}\right)$. A modulation of such dn-oidal solution describes the DSW. The slow (compared to $L$ ) evolution of the parameters of such modulation is ruled by the Whitham equations, obtained by means of Whitham averaging [40] of conservation laws of the NLSE over the period $L$. These equations are known to be expressible in the following diagonal form (due to integrability of NLSE), by introducing four Riemann invariants $r_{i}=r_{i}(t, z)$, $i=1,2,3,4, r_{1}<r_{2}<r_{3}<r_{4}$, which are a suitable combination of the original four parameters $V, \lambda_{i}$ :

$$
\frac{\partial r_{i}}{\partial z}+v_{i}\left(r_{1}, r_{2}, r_{3}, r_{4}\right) \frac{\partial r_{i}}{\partial t}=0, \quad i=1,2,3,4
$$

Here the velocities $v_{i}=v_{i}\left(r_{1}, r_{2}, r_{3}, r_{4}\right)$ constitute a deformation of the velocity $V$ that depends on combinations of $\left\{r_{i}\right\}$ and elliptic integrals of the first [i.e., $K(m)$ ] and second [i.e., $E(m)]$ kind. For instance the velocity $v_{3}=v_{3}\left(r_{1}, r_{2}, r_{3}, r_{4}\right)$ that will be relevant in the following reads as

$$
v_{3}=V-\frac{1}{2}\left(r_{4}-r_{3}\right)\left[1-\frac{\left(r_{4}-r_{2}\right) E(m)}{\left(r_{3}-r_{2}\right) K(m)}\right]^{-1},
$$

where all quantities are recast as functions of $r_{i}$, viz. $V=\frac{1}{4}\left(r_{1}+r_{2}+r_{3}+r_{4}\right)$ and $m=\left(r_{4}-r_{3}\right)\left(r_{2}-r_{1}\right) /$ $\left[\left(r_{4}-r_{2}\right)\left(r_{3}-r_{1}\right)\right]$.

Let us now recall that we are interested in describing the DSW ruled by the NLSE with initial (denoted by subscript 0 ) step-like data corresponding to $\left(\rho_{l}>\rho_{r}\right)$

$$
\begin{aligned}
& \rho_{0}= \begin{cases}\rho_{l}, & t<0 \\
\rho_{r}, & t>0,\end{cases} \\
& u_{0}= \begin{cases}0, & t<0 \\
2\left(\sqrt{\rho_{r}}-\sqrt{\rho_{l}}\right), & t>0 .\end{cases}
\end{aligned}
$$

The corresponding initial value in terms of Riemann invariants $r^{ \pm}=u \pm 2 \sqrt{\rho}$ of the unperturbed case is characterized by a constant value for $r^{-}$and a decreasing step-like variation for $r^{+}$

$$
r_{0}^{-}=-2 \sqrt{\rho_{l}}, \quad r_{0}^{+}= \begin{cases}2 \sqrt{\rho_{l}}, & t<0 \\ 4 \sqrt{\rho_{r}}-2 \sqrt{\rho_{l}}, & t>0,\end{cases}
$$

which lead, according to the dispersionless model [Eqs. (6)], in particular to a traveling (right-going) SW that connects constant states $[4,39]$. The corresponding DSW can be described in terms of a self-similar simple rarefaction wave of Whitham equations (B1) generated by the following four-dimensional initial value that arises from initial data regularization $[4,41]$ :

$$
\begin{aligned}
& r_{10}=-2 \sqrt{\rho_{l}}, \quad r_{20}=4 \sqrt{\rho_{r}}-2 \sqrt{\rho_{l}}, \quad r_{40}=2 \sqrt{\rho_{l}}, \\
& r_{30}= \begin{cases}4 \sqrt{\rho_{r}}-2 \sqrt{\rho_{l}}, & t<0 \\
2 \sqrt{\rho_{l}}, & t>0 .\end{cases}
\end{aligned}
$$

In particular, the initial value (B5) evolves in such a way that the Riemann variables $r_{1}, r_{2}, r_{4}$ remain constant and only $r_{3}=r_{3}(\zeta)$ varies, forming a pure rarefaction wave (owing to the fact that $r_{30}$ is nondecreasing) that depends on the self-similar variable $\zeta=t / z$. Indeed, all Whitham equations are formally satisfied when $r_{1,2,4}(t, z)=r_{10,20,40}$ and $r_{3}(t, z)=$ $r_{3}(\zeta)$, provided the equation $\left(\zeta-v_{3}\right) r_{3}^{\prime}=0$ is fulfilled. For $r_{3}(\zeta) \neq$ constant, this implies $\zeta=v_{3}$. The latter relation, once $v_{3}$ is expressed as $v_{3}\left(r_{10}, r_{20}, r_{3}, r_{40}\right)$ according to Eq. (B2), becomes a nonlinear equation in the only unknown $r_{3}(\zeta)$, which can be solved to find the rarefaction wave. The velocity $V_{l}$ and $V_{t}$ of the leading and trailing edges of the DSW correspond to the edges of this rarefaction wave and can be calculated as the limits of $v_{3}\left(r_{10}, r_{20}, r_{3}, r_{40}\right)$ for $r_{3} \rightarrow r_{20}$ ( $m \rightarrow 1$, soliton edge) and $r_{3} \rightarrow r_{40}(m \rightarrow 0$, linear edge), respectively. In particular for the leading edge, recalling that $r_{20}=4 \sqrt{\rho_{r}}-2 \sqrt{\rho_{l}}$, such a limit yields the result employed in Sec. IV A:

$$
\begin{aligned}
V_{l} & =\lim _{r_{3} \rightarrow 4 \sqrt{\rho_{r}}-2 \sqrt{\rho_{l}}} v_{3}\left(r_{10}, r_{20}, r_{3}, r_{40}\right) \\
& =2 \sqrt{\rho_{r}}-\sqrt{\rho_{l}}=\sqrt{\rho_{l}}+u_{r} .
\end{aligned}
$$

[1] R. Z. Sagdeev, Sov. Phys. Tech. Phys. 6, 867 (1962); R. J. Taylor, D. R. Baker, and H. Ikezi, Phys. Rev. Lett. 24, 206 (1970); A. V. Gurevich and L. P. Pitaevskii, Sov. Phys. JETP 38, 291 (1974).

[2] T. B. Benjamin and M. J. Lighthill, Proc. R. Soc. London A 224, 448 (1954); D. H. Peregrine, J. Fluid Mech. 25, 321 (1966).

[3] Z. Dutton, M. Budde, C. Slowe, and L. V. Hau, Science 293, 663 (2001); A. M. Kamchatnov, A. Gammal, and R. A. Kraenkel, Phys. Rev. A 69, 063605 (2004); R. Meppelink, S. B. Koller, J. M. Vogels, P. van der Straten, E. D. van Ooijen, N. R. Heckenberg, H. Rubinsztein-Dunlop, S. A. Haine, and M. J. Davis, ibid. 80, 043606 (2009).

[4] M. A. Hoefer, M. J. Ablowitz, I. Coddington, E. A. Cornell, P. Engels, and V. Schweikhard, Phys. Rev. A 74, 023623 (2006); J. J. Chang, P. Engels, and M. A. Hoefer, Phys. Rev. Lett. 101, 170404 (2008).

[5] J. E. Rothenberg and D. Grischkowsky, Phys. Rev. Lett. 62, 531 (1989); Y. Kodama, S. Wabnitz, and K. Tanaka, Opt.
Lett. 21, 719 (1996); C. Conti, S. Stark, P. St. J. Russell, and F. Biancalana, Phys. Rev. A 82, 013838 (2010); M. Conforti, F. Baronio, and S. Trillo, Opt. Lett. 37, 1082 (2012).

[6] W. Wan, S. Jia, and J. W. Fleischer, Nat. Phys. 3, 46 (2007); N. Ghofraniha, C. Conti, G. Ruocco, and S. Trillo, Phys. Rev. Lett. 99, 043903 (2007); S. Jia, W. Wan, and J. W. Fleischer, ibid. 99, 223901 (2007); C. Barsi, W. Wan, C. Sun, and J. W. Fleischer, Opt. Lett. 32, 2930 (2007); C. Conti, A. Fratalocchi, M. Peccianti, G. Ruocco, and S. Trillo, Phys. Rev. Lett. 102, 083902 (2009); N. Ghofraniha, L. Santamaria Amato, V. Folli, E. DelRe, S. Trillo, and C. Conti, Opt. Lett. 37, 2325 (2012).

[7] N. F. Smyth and P. E. Holloway, J. Phys. Oceanogr. 18, 947 (1988); G. A. El, H. J. Grimshaw, and A. M. Kamchatnov, Stud. Appl. Math. 114, 395 (2005); J. R. Apel, J. Phys. Oceanogr. 33, 2247 (2003).

[8] E. Bettelheim, A. G. Abanov, and P. Wiegmann, Phys. Rev. Lett. 97, 246401 (2006). 
[9] Y. C. Mo, R. A. Kishek, D. Feldman, I. Haber, B. Beaudoin, P. G. O'Shea, and J. C. T. Thangaraj, Phys. Rev. Lett. 110, 084802 (2013).

[10] N. K. Lowman and M. A. Hoefer, J. Fluid Mech. 718, 524 (2013).

[11] P. Lorenzoni and S. Paleari, Phys. D 221, 110 (2006); A. Molinari and C. Daraio, Phys. Rev. E 80, 056602 (2009).

[12] N. Ghofraniha, S. Gentilini, V. Folli, E. Del Re, and C. Conti, Phys. Rev. Lett. 109, 243902 (2012); J. Garnier, G. Xu, S. Trillo, and A. Picozzi, ibid. 111, 113902 (2013); A. Fratalocchi, A. Armaroli, and S. Trillo, Phys. Rev. A 83, 053846 (2011).

[13] P. D. Miller and Z. Xu, Commun. Pure Appl. Math. 64, 205 (2010).

[14] A. V. Gurevich and A. L. Krylov, Sov. Phys. JETP 65, 944 (1987); A. V. Gurevich, A. L. Krylov, and G. A. El, ibid. 74, 957 (1992).

[15] P. K. A. Wai, C. R. Menyuk, Y. C. Lee, and H. H. Chen, Opt. Lett. 11, 464 (1986); 12, 628 (1987); P. K. A. Wai, H. H. Chen, and Y. C. Lee, Phys. Rev. A 41, 426 (1990).

[16] N. Akhmediev and M. Karlsson, Phys. Rev. A 51, 2602 (1995).

[17] H. H. Kuehl and C. Y. Zhang, Phys. Fluids B 2, 889 (1990); V. I. Karpman and H. Schamel, Phys. Plasmas 4, 120 (1997); V. I. Karpman, Phys. Rev. E 58, 5070 (1998).

[18] V. V. Afanasjev, Y. S. Kivshar, and C. R. Menyuk, Opt. Lett. 21, 1975 (1996); C. Milian, D. V. Skryabin, and A. Ferrando, ibid. 34, 2096 (2009).

[19] I. Cristiani, R. Tediosi, L. Tartara, and V. Degiorgio, Opt. Exp. 12, 124 (2004); J. M. Dudley, G. Genty, and S. Coen, Rev. Mod. Phys. 78, 1135 (2006).

[20] D. V. Skryabin and A. V. Gorbach, Rev. Mod. Phys. 82, 1287 (2010); B. H. Chapman, J. C. Travers, S. V. Popov, A. Mussot, and A. Kudlinski, Opt. Exp. 18, 24729 (2010).

[21] A. Chabchoub, N. Hoffmann, M. Onorato, G. Genty, J. M. Dudley, and N. Akhmediev, Phys. Rev. Lett. 111, 054104 (2013).

[22] S. P. Stark, A. Podlipensky, and P. St. J. Russell, Phys. Rev. Lett. 106, 083903 (2011).

[23] N. Y. Joly, J. Nold, W. Chang, P. Hölzer, A. Nazarkin, G. K. L. Wong, F. Biancalana, and P. St. J. Russell, Phys. Rev. Lett. 106, 203901 (2011); M. F. Saleh, W. Chang, P. Hölzer, A. Nazarkin, J. C. Travers, N. Y. Joly, P. St. J. Russell, and F. Biancalana, ibid. 107, 203902 (2011).

[24] M. Erkintalo, Y. Q. Xu, S. G. Murdoch, J. M. Dudley, and G. Genty, Phys. Rev. Lett. 109, 223904 (2012).

[25] P. Colman, S. Combrié, G. Lehoucq, A. de Rossi, and S. Trillo, Phys. Rev. Lett. 109, 093901 (2012).

[26] B. B. Zhou, A. Chong, F. W. Wise, and M. Bache, Phys. Rev. Lett. 109, 043902 (2012); M. Conforti and F. Baronio, J. Opt. Soc. Am. B 30, 1041 (2013); M. Conforti, F. Baronio, and C. De Angelis, IEEE Photonics J. 2, 600 (2010).
[27] E. Rubino, J. McLenaghan, S. C. Kehr, F. Belgiorno, D. Townsend, S. Rohr, C. E. Kuklewicz, U. Leonhardt, F. König, and D. Faccio, Phys. Rev. Lett. 108, 253901 (2012); M. Conforti, N. Westerberg, F. Baronio, S. Trillo, and D. Faccio, Phys. Rev. A 88, 013829 (2013).

[28] HOD terms can be relevant also for spatial problems to account for nonparaxial effects. See, e.g., C. Conti, G. Ruocco, and S. Trillo, Phys. Rev. Lett. 95, 183902 (2005).

[29] Y. Liu, H. Tu, and S. A. Boppart, Opt. Lett. 37, 2172 (2012); L. E. Hooper, P. J. Mosley, A. C. Muir, W. J. Wadsworth, and J. C. Knight, Opt. Express 19, 4902 (2011); A. M. Heidt, A. Hartung, G. W. Bosman, P. Krok, E. G. Rohwer, H. Schwoerer, and H. Bartelt, ibid. 19, 3775 (2011); C. Finot, B. Kibler, L. Provost, and S. Wabnitz, J. Opt. Soc. Am. B 25, 1938 (2008).

[30] K. E. Webb, Y. Q. Xu, M. Erkintalo, and S. G. Murdoch, Opt. Lett. 38, 151 (2013).

[31] M. Conforti and S. Trillo, Opt. Lett. 38, 3815 (2013).

[32] The nonlinear contribution to the wave number of the resonant radiation is induced by cross-phase modulation with a nonzero background, on top of which RR propagates.

[33] G. B. Whitham, Linear and Nonlinear Waves (Wiley, New York, 1974).

[34] In the intermediate regime $0.5<\left|\beta_{3}\right|<1$, an estimate of the leading-edge velocity could be given via a nonintegrable formulation of the Whitham modulation theory, see G. A. El, Chaos 15, 037103 (2005) and Ref. [10], which, however, requires further studies.

[35] In the framework of different contexts and models, it was conjectured that a similar phenomena could be a specific manifestation of nonintegrability in dispersive dissipationless systems. See G. A. El, H. J. Grimshaw, and N. F. Smyth, Phys. Fluids 18, 027104 (2006); G. A. El, A. Gammal, E. G. Khamis, R. A. Kraenkel, and A. M. Kamchatnov, Phys. Rev. A 76, 053813 (2007).

[36] J. C. DiFranco, P. D. Miller, and B. K. Muite, Acta Math. Scientia 31B, 2343 (2011).

[37] M. Conforti, F. Baronio, and S. Trillo, Opt. Lett. 38, 1648 (2013).

[38] S. B. Cavalcanti, J. C. Cressoni, H. R. da Cruz, and A. S. Gouveia-Neto, Phys. Rev. A 43, 6162 (1991); S. Pitois and G. Millot, Opt. Commun. 226, 415 (2003); J. D. Harvey, R. Leonhardt, S. Coen, G. K. L. Wong, J. C. Knight, W. J. Wadsworth, and P. St. J. Russell, Opt. Lett. 28, 2225 (2003).

[39] R. J. LeVeque, Finite-Volume Methods for Hyperbolic Problems (Cambridge University Press, Cambridge, 2004).

[40] G. B. Whitham, Proc. R. Soc. London, Ser. A 283, A238 (1965).

[41] Y. Kodama, SIAM J. Appl. Math. 59, 2162 (1999). 